\title{
Extracorporeal Reconstruction of the Radial Head
}

Kow Ren Yi ${ }^{1,2}$, Zaharul Azri Mustapha Zakaria3 ${ }^{3}$ Ruben Jaya Kumar ${ }^{3}$, Low Chooi Leng ${ }^{4}$, Khairul Nizam Siron Baharom²

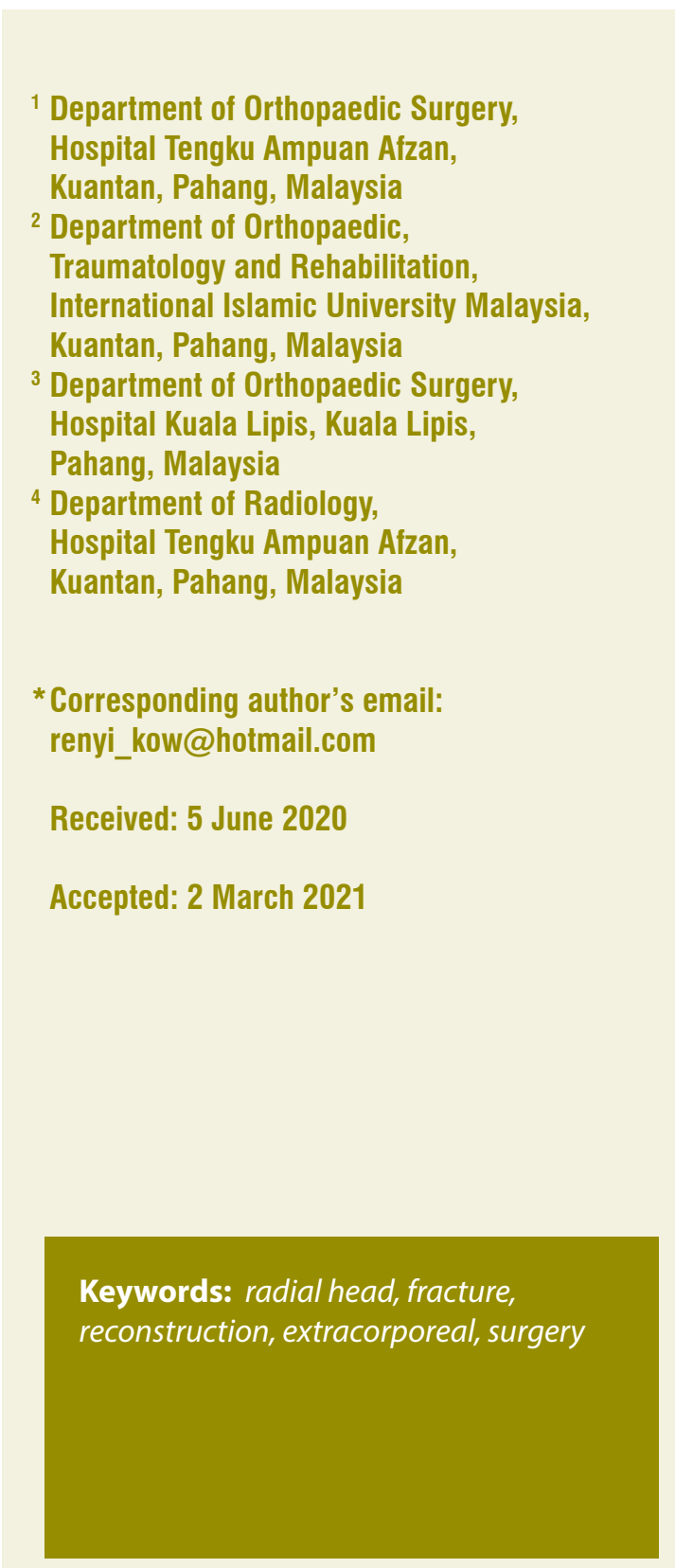

\section{ABSTRACT}

Radial head fractures are common in adults. The radial head acts as an important stabilizer of the radius, especially during the forearm pronation and supination. Surgical treatment of Mason type III fracture of the radial head includes radial head excision, radial head replacement and open reduction and internal fixation. For patients treated with radial head excision, the radius might migrate proximally, causing complications such as chronic pain at the wrist, forearm, and elbow. On the other hand, radial head arthroplasty is associated with a high revision and removal rate. Hence, open reduction and internal fixation are preferred when feasible. This is technically demanding due to the severe displacement of the radial head fragments and limited surgical space. Mr K, a 30-year-old manual worker sustained a closed right radial head fracture Mason type III after an alleged fall. The radial head was displaced anteriorly. Intra-operatively, the radial headpieces were retrieved and were reconstructed extracorporeal and fixed with a miniplate. The patient subsequently recovered with good function. The new technique of extracorporeal reconstruction of the radial head offers a viable option to the surgeon with similar, or even better, outcomes in terms of elbow function.

\section{INTRODUCTION}

Radial head fracture is the most common fracture at the elbow in adults (Pike et al., 2009). It commonly results from a fall on an outstretched hand (Kumar et al., 2015). In 1954, Mason reviewed one hundred cases of 
radial head fractures and classified them into 3 types. According to Mason, type I radial head fractures are non-displaced or they involve only the peripheral rim; type II fractures involve the displacement of the marginal sector; and type III fractures are comminuted and displaced radial head fractures (Mason, 1954).

The head of the radius is important for stabilization of the radius during pronation and supination of the forearm. Besides that, the radial head also acts as a fulcrum for transmission of the axial forces across the elbow joint. In Mason type III fracture of the radial head, removal of the fragments of the radial head is an option as it does not alter the elbow biomechanics. Nevertheless, this procedure comes with risks of developing degenerative changes, valgus instability or early osteoarthritis of the ulnohumeral joint. Hence, the current trend is moving towards open reduction and internal fixation (ORIF) of the radial head to restore the alignment and stability of the elbow joint (Yu et al., 2016). Owing to the limited surgical space, in vivo reconstruction of the radial head might be technically demanding. Extracorporeal reconstruction, where the radial head is recovered and reconstructed outside the body, and then reinserted back into the body, has shown promising results (Kumar et al., 2015; Yu et al., 2016). We present a case of Mason type III radial head fracture which was successfully treated with this method.

\section{CASE PRESENTATION}

Mr KRL, a 30-year-old right hand dominant manual worker with no known medical illness, was involved in an industrial injury in which he fell from a 5-metre height and landed on his outstretched right hand. He presented with pain and swelling at his right elbow with limited right elbow range of movement due to the pain. He did not complain of pain in his right shoulder or wrist and he had no other associated injury. Plain radiographs (anteroposterior and lateral views) of his right elbow showed Mason type III radial head fracture which was confirmed intra-operatively by the intensifying imaging (Figure 1). There was no dislocation of the elbow joint and the ulnar coronoid process was intact.

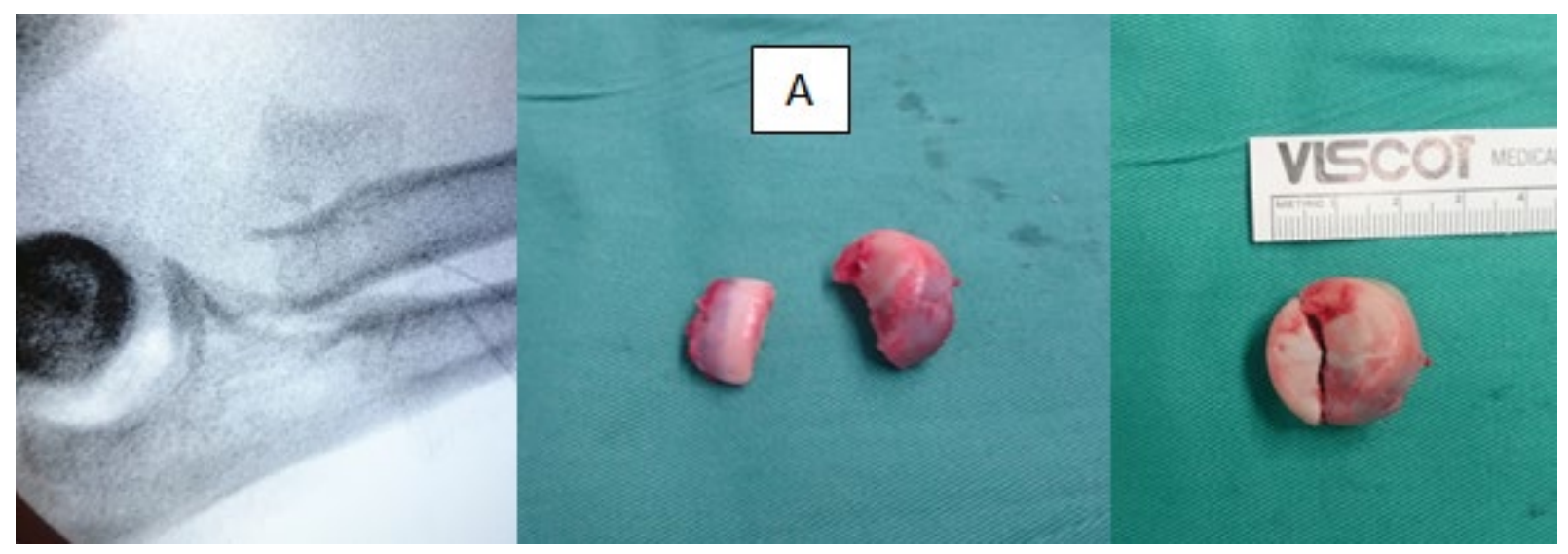

Figure 1 (A) An intra-operative intensifying image of the Mason type III radial head fracture and the radial head after removal. Even though the radial head is not severely comminuted, it is severely displaced anteriorly, making in vivo radial head reconstruction impossible. A small anterior incision is made at the antecubital fossa to facilitate the retrieval of the radial head. 
Since the patient was young, productive, and right hand dominant, we decided to reconstruct the right radial head to avoid radial head resection or arthroplasty. The attempt to perform reduction and fixation in vivo was futile due to the severe anterior displacement of the radial head fragments. We attempted to reduce the radial head fragment with a Kirschner wire but failed to owe to the fragment trapped in the soft tissues. Hence, the radial head fragments were retrieved anteriorly, and an extracorporeal reconstruction was done (Figure 2). The radial head fragments were temporarily held with a Kirschner wire on a mini T-plate before fixation with screws (Figure 2). The reconstructed radial head with a mini T-plate was then transferred to the fracture site and its distal site was fixed to the radial shaft (Figure 3). The partially torn annular ligament was repaired accordingly after fixation of the radial head. Before closure, the elbow was examined for any instability.

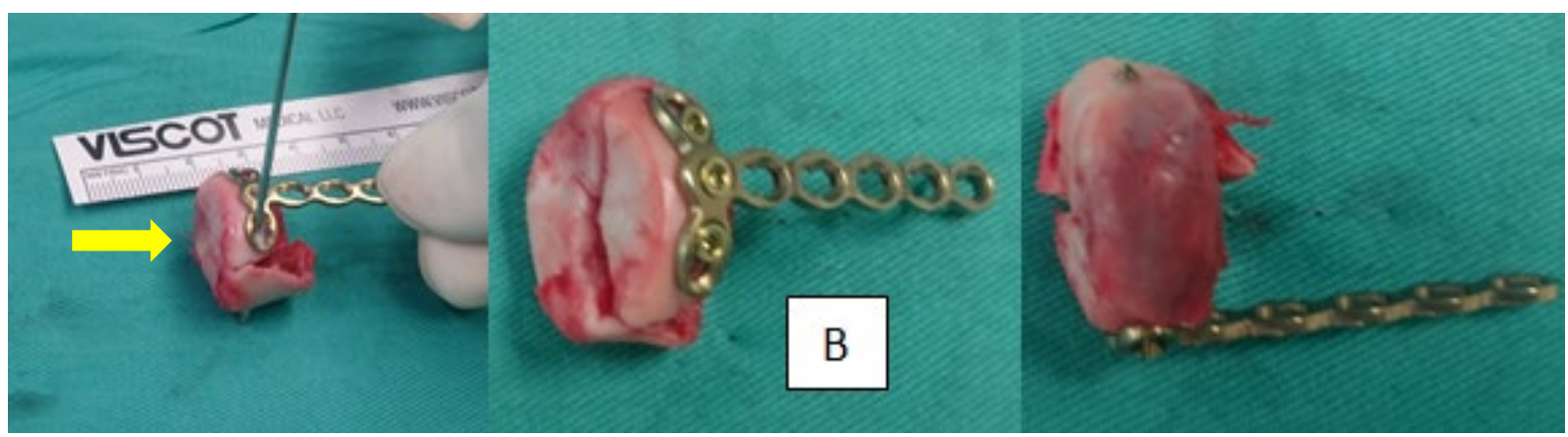

Figure 2 (B) The extracorporeal method of reconstruction of the radial head. The mini T-plate is fixed to the radial head with a temporary Kirschner wire (yellow arrow) before fixation with screws.

Post-operatively, his right elbow was protected with a back slab for 3 weeks and then he was started on active range of movement exercises as tolerated. Bony union was achieved at three months post-operation (Figure 4) with a good outcome based on the Mayo Elbow Performance Score (MEPS -80 ) and he was able to return to work. There was no complication at 6-months and 1-year follow-up respectively. At the last follow-up, the elbow range of motion of this patient was $10-100^{\circ}$, with $45^{\circ}$ pronation and $30^{\circ}$ supination. We were not able to report the long-term outcome of this patient as the patient had returned to his home country and attempts to contact him were futile.

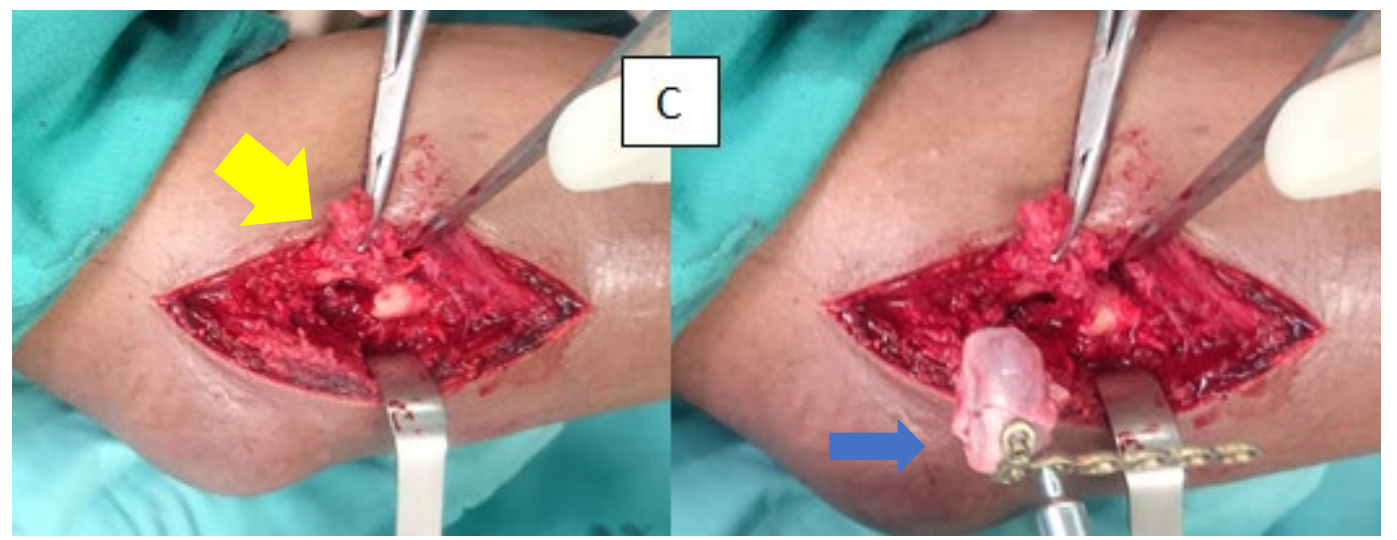

Figure 3 (C) The Kocher approach (yellow arrow) to retrieve the radial head and the position of the radial head (blue arrow) before definitive fixation. 


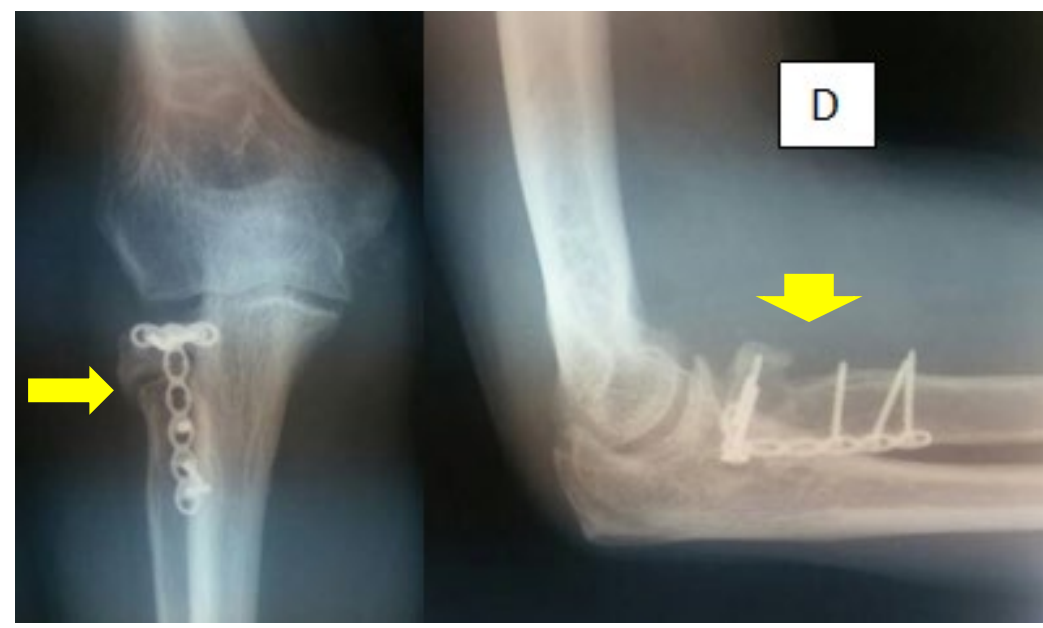

Figure 4 (D)

Bony union on the plain radiographs (yellow arrow) (anteroposterior and lateral views) with good outcomes and the patient could return to work three months after the surgery.

\section{DISCUSSION}

Radial head excision is an accepted form of treatment for a comminuted fracture such as Mason type III fracture (Li et al., 2014; VanBeek et al., 2010). As compared to conservative management or ORIF, this method of treatment has produced better outcomes in some studies (Ikeda et al., 2003). Nevertheless, this mode of treatment comes with a risk as the loss of the radial head might cause proximal radial migration, leading to pain at the wrist, forearm, and elbow (Ikeda et al., 2003). Furthermore, the loss of radiocapitellar contact leads to the development of ulnohumeral arthrosis, weakness, and instability (Kodde et al., 2015; Kumar et al., 2015). To maintain the congruity of the proximal radioulnar joint, several authors recommend the preservation and fixation of the radial head, thereby retain the kinematics and stability of the elbow joint (Ikeda et al., 2005; Kodde et al., 2015). This can be achieved by internal fixation using mini plates or Herbert screws, even in the event of severely displaced or comminuted fracture (Ikeda et al., 2003). Ikeda et al reported patients with comminuted radial head fractures who underwent ORIF achieved a better outcome in terms of joint movement, elbow power and functional score compared to patients who underwent radial head resection (Ikeda et al., 2005).
A biomechanical study shows that fixation with a mini T-plate is more superior compared to a radial head replacement (RHR), in terms of withstanding rotational forces. Radial head replacement is also associated with high removal or revision rate following RHR due to complex radial head fractures ( $\mathrm{Yu}$ et al., 2016). While the functional outcomes of RHR and ORIF are similar, the cost of performing an RHR is significantly higher than ORIF (Al-Burdeni et al., 2015). On top of that, up to $26 \%$ of the patients who underwent RHR subsequently developed heterotopic ossification (Tarallo et al., 2017).

Open reduction and internal fixation for Mason type III radial head fractures can be technically demanding as some fragments can be severely displaced and the operating surgical field is limited. This problem is solved by the "on-table reconstruction of the radial head" technique in which all the radial head fragments are retrieved and are reconstructed on-table (Businger et al., 2010; Kumar et al., 2015). A Kirschner wire is inserted to temporarily hold the radial head fragments before fixation to the mini T-plate. The mini T-shaped plate is the implant of choice for this type of fracture as the radial head fragments can all be fixed to the proximal part of the plate. The reconstructed radial head with the miniplate is then transferred to the fracture site and are anchored with distal screws. This technique has been used by several authors 
with good outcomes in their series of patients (Businger et al., 2010; Kumar et al., 2015). The reported risk of this technique includes nonunion, secondary fragment displacement and heterotopic ossification, but thus far there is no reported incidence of infection or implant failure. In a case series by Businger et al. (2010), where they reconstructed the radial head using this technique, there were no nonunion or avascular necrosis reported among the six patients operated (Businger et al., 2010). Nevertheless, in a case series of 6 patients by Kumar et al, half of them ( 3 patients) had nonunion, including one case of avascular necrosis of the radial head (Kumar et al., 2015). The authors postulated that preoperative metaphyseal bone loss, coupled with comminuted radial head fracture, was the cause of non-union and avascular necrosis of the radial head (Kumar et al., 2015). In the event of non-union of the radial head, the patient's functional status will not be affected. Among 326 patients reviewed by Kang et al, 6 patients $(1.8 \%)$ suffered from nonunion of the radial head, and all of them achieved satisfactory clinical outcomes after an average follow-up of 7.6 years (Kang et al., 2012).

We believe that this technique of ORIF is functionally superior to radial head excision and more cost-effective than radial head replacement. In our patient, he recovered with a good outcome and he was able to return to manual work. The technique used in this case report is limited by a relatively short-term outcome (up to 1 year) and the long-term outcome is yet to be determined.

\section{CONCLUSION}

There are various surgical interventions for Mason type III radial head fractures, including excision of radial head, radial head replacement and open reduction and internal fixation. Via extracorporeal approach, an open reduction and internal fixation of the radial head is feasible for this type of injury.

\section{CONFLICT OF INTEREST}

The authors declare that they have no competing interests in publishing this case.

\section{CONSENT}

Written consent was obtained from the patient to publish the case. A copy of the written consent is available for review by the Chief Editor.

\section{ACKNOWLEDGEMENTS}

This publication is supported by SASMEC @ IIUM Research Grant (SRG).

\section{REFERENCES}

Al-Burdeni, S., Abuodeh, Y., Ibrahim, T., \& Ahmed, G. (2015). Open reduction and internal fixation versus radial head arthroplasty in the treatment of adult closed comminuted radial head fractures (Modified Mason Type III and IV). International Orthopaedics, 39 (8), 1659 - 1664. https://doi.org/10.1007/ s00264-015-2755-1.

Businger, A., Ruedi, T. P., \& Sommer, C. (2010). On-table reconstruction of comminuted fractures of the radial head. Injury, 41 (6), 583 - 588. https://doi.org/10.1016/j. injury.2009.10.026.

Ikeda, M., Yamashina, Y., Kamimoto, M., \& Oka, Y. (2003). Open reduction and internal fixation of comminuted fractures of the radial head using low-profile mini-plates. The Journal of Bone and Joint Surgery, 85 (7), 1040 - 1044. https://doi.org/10.1302/0301620x.85b7.13823.

Ikeda, M., Sugiyama, K., Kang, C., Takagaki, T., \& Oka, Y. (2005). Comminuted fractures of the radial head: Comparison of resection and internal fixation. The Journal of Bone and Joint Surgery, 87 (1), 76 - 84. https://doi. org/10.2106/JBJS.C.01323.

Kang, H. J., Shin, S. J., \& Kang, S. S. (2012). Nonunion of the radial neck following operative treatment for displaced radial head and neck fractures. Acta Orthopaedica Belgica, 78, 597 - 602. PMID: 23162955. 
Kodde, I. F., Kaas, L., Flipsen, M., van den Bekerom, M. P. J., \& Eygendaal, D. (2015). Current concepts in the management of radial head fractures. World Journal of Orthopedics, 6 (11), 954 - 960. https://doi.org/10.5312/wjo. v6.i11.954.

Kumar, G. N. K., Sharma, G., Farooque, K., Sharma, V., Jain, V., Singh, R., \& Morey, V. (2015). Ontable reconstruction and fixation of Mason type III radial head fractures. Chinese Journal of Traumatology, 18 (5), 288 - 292. https:// doi.org/10.1016/j.cjtee.2015.11.005.

Li, N., \& Chen, S. (2014). Open reduction and internalfixation versus radial head replacement in treatment of Mason type III radial head fractures. European Journal of Orthopaedic Surgery \& Traumatology, 24 (6), 851 - 855. https://doi.org/10.1007/s00590-013-1367-y.

Mason, M. L. (1954). Some observations on fractures of the head of the radius with a review of one hundred cases. British Journal of Surgery, 42 (172), 123 - 132. https://doi.org/10.1002/ bjs. 18004217203 .
Pike, J. M., Athwal, G. S., Faber, K. J., \& King, G. J. W. (2009). Radial head fractures - an update. Journal of Hand Surgery, 34 (3), 557 - 565. https://doi.org/10.1016/j.jhsa.2008.12.024.

Tarallo, L., Mugnai, R., Rocchi, M., Capra, F., \& Catani, F. (2017). Mason Type III radial head fractures treated by anatomic radial head arthroplasty: Is this a safe treatment option? Orthopaedics \& Traumatology: Surgery \& Research, 103 (2), 183 - 189. https://doi. org/10.1016/j.otsr.2016.10.017.

VanBeek, C., \& Levine, W. N. (2010). Radial HeadResect, Fix or Replace. Operative Technique in Orthopaedics, 20(1), 2-10. https://doi. org/10.1053/j.oto.2009.08.003.

Yu, W., Hu, J., Zhang, X., Zhu, X., Xu, Y., Yi, J., \& Liu, Y. (2016). Acute unstable complex radial head and neck fractures fixed with a mini T-shaped plate in a 20-year-old man: a case report. Therapeutics and Clinical Risk Management, 12, 825-830. https://doi.org/10.2147/TCRM. S107640. 